\title{
Fitting of Constrained Feature Models to Poor 3D Data
}

\author{
C. Robertson, R. B. Fisher, N. Werghi, A. P. Ashbrook \\ Machine Vision Unit, Institute for Perception, Action and Behaviour, \\ Division of Informatics, University of Edinburgh, \\ Edinburgh, EH1 2QL, UK \\ craigr@dai.ed.ac.uk
}

\begin{abstract}
In this work we have addressed the question of whether it is possible to extract parametric models of features from poor quality $3 D$ data. In doing so we have examined the applicability of an evolutionary strategy to the problem of fitting constrained parametric models. In the first phase, a background surface is fitted and removed leaving points of discontinuity associated with the feature. Then the discontinuities are classified, using the RANSAC algorithm, into drilled hole artifacts or blade edges suggesting drilled slots. This information, as well as the set of discontinuity points is passed to the Genocop III algorithm, proposed by Michalewicz [3], for optimization using a priori geometric constraints. Results, example times for convergence and comparisons with known ground truths are given.
\end{abstract}

Keywords: Model fitting, Ransac, evolutionary algorithms.

\section{Introduction}

Shape analysis of objects from range data (captured three dimensional coordinates of surface points) is a key problem in computer vision with several important applications in manufacturing, such as assembly, quality control and reverse-engineering. In this work we have examined whether it is possible to extract parametric models of features from poor quality 3D data. This kind of reconstruction problem is generally formulated as a nonlinear programming problem (NLP), which tries to optimally fit the data to candidate shape descriptions. The NLP optimises a function subject to several constraining equations and inequalities. Especially with nonlinear constraints, it is notoriously difficult to optimise and there is no known method to guarantee a satisfactory solution. Traditional search techniques, such as gradient descent, are unsatisfactory for the solution of NLPs, due to the local nature of their search methods and the 
reliance on smooth derivatives in the search-space. In previous work $[8,9]$ we examined the applicability of evolutionary strategies to the problem of fitting constrained lines, planes and degenerate second order surface to both synthetic and acquired object range data. In this paper we examine the applicability of fitting parametric models to poor quality range data from small complex artifacts such as drilled holes and machined slots of differing cross-section. The GenocopIII algorithm developed by Michalewicz [3], Ch.7 has been used and extended in this paper by adding a complex model fitting function. It is an evolutionary algorithm which is specialised to handle constrained function optimisation and particular to it is the handling of non-linear constraints. It uses real-valued genes and includes methods to deal with linear, non-linear, domain and inequality constraints. We have used a specialised fitness function (described in section 3.5), which is applied to the problem of fitting parametric 3-dimensional model chromosomes to range data while simultaneously applying several necessary constraints. The constraints applied are of two types : domain (the restriction on the parameter size) and relational (relationships between surfaces that are known a priori).

Since this problem has a specific context it is important to illustrate it. Our group is researching the reverse engineering of machined parts. These parts are often complex and possess many surfaces which may have known geometric relationships. Segmentation and parameterisation of the captured 3-dimensional range data is a difficult multi-part task involving the following elements:

1. Data collection. This is performed using a moving-bed, orthogonal laser stripe ranger which provides data at up to $0.5 \mathrm{~mm}$ steps in the $\mathrm{X}-\mathrm{Y}$ plane. Noise on the data is around $0.15 \mathrm{~mm}$ standard deviation.

2. Data registration. This is performed using a variant of the iterated closest point algorithm [1].

3. Segmentation. There are many ways of segmenting the $3 \mathrm{~d}$ dataset, most are based upon changes in local surface curvature followed by some form of least-squares optimisation, for example [4].

4. Exploitation of constraints. Constraints may be applied to exploit knowledge about surface relationships.

The formulation of constraints and the application of constraint-based correction and optimisation of surface fitting has been achieved previously [2] with notable success using the several constraint application strategies. There are, however, some associated problems with this approach: complex formulation of the constraint function; heavy reliance on the global convexity of the solution space; reliance on accurate initial estimate of solution.

The 'processing pipeline' that is required for this approach also can lead to a build-up of problems that must be solved in the constraint application stage. Previously $[8,9]$ it was demonstrated that simultaneous fitting and constraint 


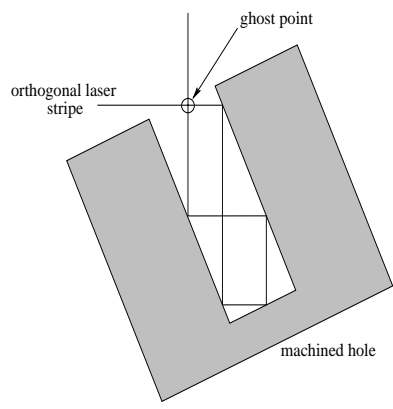

(a) Reflection problems

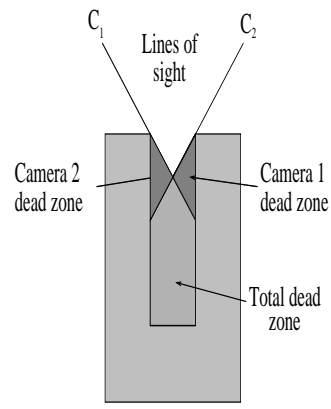

(b) Dead zone problems

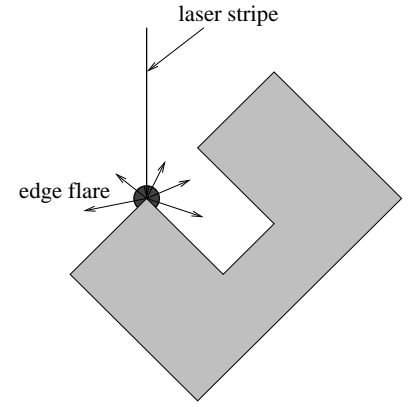

(c) Edge Flare

Figure 1: Laser ranger problems

management using all the available data could be achieved by a single evolutionary algorithm using careful chromosome management and good generation of starting conditions.

In this paper we address the fitting of complex models of artifacts such as slots and drilled holes which are often found in machined mechanical parts. There are, however, several problems unique to this fitting, stemming from the size of the artifacts and the nature of the machined surfaces from which they are made.

\section{Method}

\subsection{Data Quality and Sampling Issues}

The sampling density of the range data is $0.5 \mathrm{~mm}$ in the $\mathrm{X}$ and $\mathrm{Y}$ dimensions. This is a good density for the fitting of large convex surfaces, often of around $100 \mathrm{~cm}^{2}$ area, where the samples can easily be accumulated to build a very accurate model. However, when gathering data from drilled holes or slots it is the points of discontinuity that are the important points. The parametric fitting of the supporting surface is relatively straightforward and leaves the complex mass of data representing the artifacts (see figure 2). Reflections from the inside walls of the artifact also cause problems because the machined surface is highly reflective and often has a shape that amplifies this. This causes ghost points that although consistent between two viewing cameras, are anomalous, as shown in figure 1(a). Camera dead-zones also cause data to be lost from the bottom of both drilled holes and machined slots when a camera can no longer track the laser stripe, figure 1(b). There is a further complicating factor in finding artifacts which is due to laser specularities around points of rapid discontinuity such as the edges of holes or slots. These specularities (edge flare) are due to the finite width of the laser which becomes split at the discontinuity. Although local this can mean that the edges of such artifacts are very difficult to accurately ascertain, as shown in figure $1(\mathrm{c})$. 


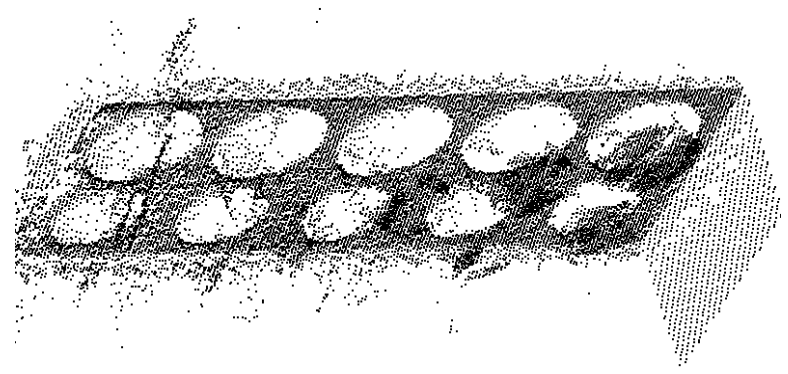

Figure 2: Typical poor data
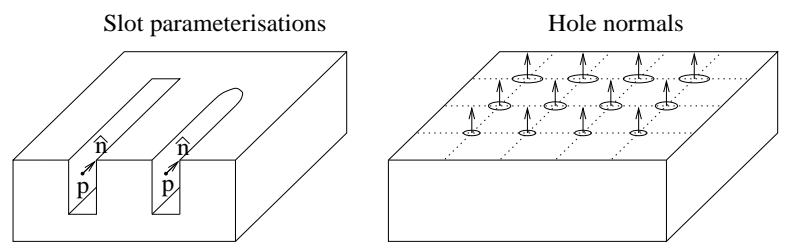

Figure 3: Artifact Models

Data (plotted as XYZ points) which shows many of these problems are shown in figure 2 .

\subsection{Primitives and Models}

Our feature models have only three basic primitives:

1. Straight edges, where planes in the data intersect.

2. Curves edges, where machined holes or curved slots end.

3. Planes, which are often the supporting surfaces of the machined artifacts as well as the bases of slots and holes.

The three models we have experimented with are :

- Orthogonal drilled hole : Circular surface discontinuity and a plane defining the bottom of the hole.

- Square ended slot : Represented by three or four lines of discontinuity defining its edges and a plane defining the bottom.

- Cylindrical ended slot : Represented by two lines of discontinuity defining its edges, one or two semi-circular endings and a plane defining the bottom.

Although there are many other possible models we have used these to demonstrate the principle. Further to the basic models, our optimization uses geometric relationships between the models such as colinearity of drilled holes, slots with the same axis normal and distance relationships. 


\subsection{Full Models}

For reconstruction, definitions of full models are required, which consistin of primitive collections and their relationships to each other. In our case we have built models only by the application of constraints at the optimization stage. For example, since a slotted part is characterized by planes intersecting at various angles, we use a model of this only using those angular constraints, adding distance constraints when they are known. Examples of models used in this work are given below:

- Cylindrical slotted part. This part has three machined slots at different depths. The model of this consists of one unit vector normal, describing the slot direction, three 3D points describing the positions of the slots and one parameter specifying the slot widths. The parameterisation has a priori constraints that: the slots are the same width; the slots are all lying in the same direction; the slot widths also define the estimate of the diameter of the machine tool used.

- Square slotted part. This part also has three machined slots at different depths. The model of this consists of one unit vector normal, describing the slot direction, three 3D points describing the positions of the slots and one parameter specifying the slot widths. The parameterisation has $a$ priori constraints that: the slots are the same width; the slots are all lying in the same direction; the end planes are lying along the same direction.

- Drilled hole part 1. This part consists of two sets of five holes drilled at different depths and radii. Each set of holes is constrained to be co-linear and hole radii of each set constrained to be equal. One set has a radius of seven millimetres, the other is nine millimetres. Hole spacing is a free variable. See figure 3(a).

- Drilled hole part 2. This part consists of three sets of holes drilled at different depths and radii. Each set of holes is constrained to be co-linear with the hole radii of each set constrained to be equal. The radii are : (5x) $5 \mathrm{~mm},(4 \mathrm{x}) 3 \mathrm{~mm},(4 \mathrm{x}) 4 \mathrm{~mm}$. Number of holes at that radius are shown in brackets. Each set of holes is constrained to be co-linear and radii of each set constrained to be equal. See figure 3(b).

- Compound slot part. This part consists of four slots, two square-ended, two round ended machined into the same block. The square slots have one square closed end, one open end. This is repeated with the same for the round ended slots. The parameterisation has a priori constraints that: the slots are the same width; the slots are all lying in the same direction; the end planes are lying along the same direction.

These models are encoded implicitly inside the optimization function as explained in section 3.2. 


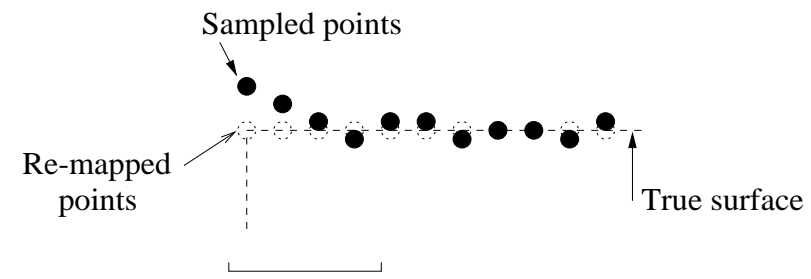

Edge flare effect

Figure 4: Discontinuity re-mapping to reduce edge flare

\section{Algorithm}

Our algorithm consists of three serial processes :

1. Extraction of primitives using the Ransac algorithm [11].

2. Heuristic identification of models present.

3. Model fit optimisation using the Genocop III algorithm [3] using geometric constraints.

\subsection{Primitive Extraction}

Supporting surfaces were extracted from the dense range data using a Ransac plane finder [11]. Sub-pixel positions of discontinuities on these surfaces were then found by applying a local gradient operator and re-projecting the discontinuities back to the supporting surface. This has the effect of reducing the edge flare effects quite substantially as shown in figure 4 .

Once a set of discontinuities has been found, a composite Ransac line and circle finder was used to extract likely primitives together with their parameterisations. Example images of the range data, segmentation and primitive extraction are shown in figure $5(\mathrm{a}-\mathrm{d})$.

Once primitives have been extracted, when appropriate, the point sets and parameterisations are passed to an evolutionary algorithm for further optimisation using geometric constraints. The details of this algorithm are discussed below.

\subsection{Model Formulation}

In standard GAs binary encoding forms the chromosomes in the solution, however in an evolution program each gene is a floating point number. Genes are then concatenated into a single chromosome.

In the case of lines on the support plane, we have used the 4 gene parametric representation : $A:<\hat{n}, d>$ where $\hat{n}$ is the unit normal describing the line and $d$ is the constant defining its minimum distance from the origin. In the case of circles,also in the support plane, we have used the 4 gene parametric 


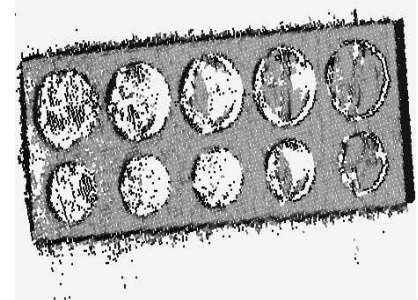

a) Range data (cosine shaded)

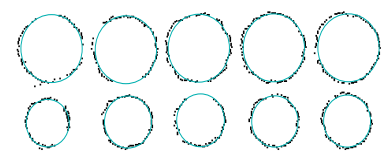

c) Derived primitives

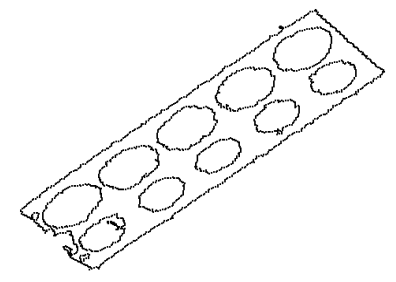

b) Discontinuities

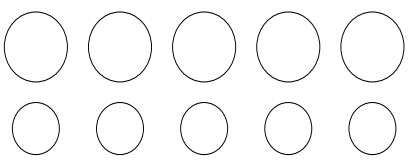

d) Geometric constraints applied

Figure 5: Primitive Extraction Process

representation : $A:<\mathbf{P}, r>$ where $\mathbf{P}$ is the centre point and $r$ is the radius. The normal is inherent in the supporting plane direction.

A full chromosome, $G$, describing a given artifact set, is represented by a set of concatenated part-chromosomes: $G=\left\{A_{j}\right\}$. This representation of a set of artifacts as a chromosome is much shorter than, say, a set of general quadrics as explored in [8]. This cuts down the complexity of constraints and makes it amenable to straighforward manipulation without the need to employ constraints on the forms themselves, only on sets of forms taken as systems. Since many of the parameter constraints are implicit, the parameters can be optimised under just a handful of constaints (mostly on their domains) which is relatively cheap computationally.

\subsection{Genocop II and III}

Calculus based methods assume that the objective function, $f(\mathbf{x})$, and all constraints are continuously differentiable functions of $\mathbf{x}$. The general approach is to transform the non-linear problem $(N L P)$ into a sequence of sub-problems and then solve those, requiring an explicit computation of the objective function. Some of these methods become ill-conditioned and fail.

Genocop II uses a sequential quadratic penalty function and is formulated as the optimisation of the function:

$F(\mathbf{x}, r)=f(\mathbf{x})+\frac{1}{2 r} \bar{C}^{T} \bar{C}$, where $r>0, f$ is the user defined evaluation function and $\bar{C}$ is the vector of active constraints, $c_{1}, \ldots, c_{l}$.

Attia has provided solutions to the instability of this approach [10]. The set of all constraints, $C$, is divided into the linear constraints, $L$, the non-linear equations, $N_{e}$ and the non-linear inequalities, $N_{i}$. A set of active constraints, $A$ is then built from $N_{e}$ and the violated constraints from $N_{i}$ (a constraint is said to be violated if it is more than some tolerance $\delta$ from its correct value), 
which are called $V$. The structure of Genocop II is outlined in [3]. Inside its main loop, Genocop I optimizes the function $F(\mathbf{x}, r)=f(\mathbf{x})+\frac{1}{2 r} \bar{A}^{T} \bar{A}$. Several mutation operators take an initially identical population and introduce diversity to it. At convergence, the best individual, $\mathbf{x}^{*}$, is saved and the the value of the penalty parameter is decreased.

Most of the essential elements of Genocop III are the same as those of Genocop II. However, in this algorithm two populations are kept, a reference set $\bar{R}$ and a search set $\bar{S}$. The reference population is a set of fully feasible individuals which satisfy all the constraints whereas the search population may not. At each iteration, the search population are allowed to move around the solution space and are repaired back onto the constraint manifold. If the search point is $\bar{S}$ and the reference point is $\bar{R}$, then a random point $\bar{Z}$ is created from the segment between $\bar{S}$ and $\bar{R}$ by generating a value, $a \in[0,1]$ then :

$$
\bar{Z}=a \bar{S}+(1-a) \bar{R}
$$

Once a feasible $\bar{Z}$ is found, if it is better than $\bar{R}$, then that reference point is replaced with some probability. As the iterations progress, the set of reference points converge to the maximum or minimum on the search space.

\subsection{Evaluation Function and Point Assignment for the Fitting}

In our application of Genocop III the evaluation function is novel in that it is a multi-objective optimization. For each point, $x_{i}$, the true geometric distance to the theoretical primitive is computed and this is used as the least-squares error for that point relative to that primitive.

$$
e_{i}=\min _{p}\left\{\operatorname{dist}\left(\mathbf{x}_{i}, S_{p}\right)\right\}
$$

where $e_{i}$ is the error for the point $\mathbf{x}_{i}, p$ is the index of $M$ theoretical primitives, $i$ is the index of $N$ points, $S_{p}$ is the parameterised primitive and dist is the distance to that primitive.

The evaluation function to be minimised is then the sum of these minima :

$$
E=\sum_{i=0}^{i=N} e_{i}
$$

\subsection{Starting Conditions and Relational Constraints}

In virtually all cases, domain constraints on individual genes are used to narrow the search space for that gene. These are represented as one permanent part of the sequential quadratic penalty function matrix [3] used in the evaluation function. A good example of where domain constraints can reduce the search space is in the case of the three parameters describing a unit normal. Each of these parameters can never be outside the range $[-1,+1]$ so these make good domain constraints. 
In-chromosome relational constraints are straightforward to formulate when a parametric form is used. For example, consider two planes:

$P_{1}=<n_{x 1}, n_{y 1}, n_{z 1}, d_{1}>$ and

$P_{2}=<n_{x 2}, n_{y 2}, n_{z 2}, d_{2}>$

which are known a priori to be orientated orthogonally. In this case, the chromosome would have the form :

$G=\left\{n_{x 1}, n_{y 1}, n_{z 1}, d_{1}, n_{x 2}, n_{y 2}, n_{z 2}, d_{2}\right\}$

and the orthogonality constraint would then appear as a non-linear inequality of the form: $\left(n_{x 1} * n_{x 2}\right)+\left(n_{y 1} * n_{y 2}\right)+\left(n_{z 1} * n_{z 2}\right) \leq \epsilon$ where $\epsilon$ is the constraint tolerance value.

In order to perform the optimisation, Genocop requires a starting position on the constraint manifold. This is the seed for the search points which are then mutated around it. It is also used to produce the set of reference points as described earlier in section 3.4. In our case this means designing a chromosome which is both close to being a concatenation of the individual least-squares results for the part-chromosomes as well as fulfilling the domain and relational constraints. Starting conditions for increasingly complex solutions with increasingly complex constraints have previously been found to be difficult [8] for the general quadric. However, when a parametric representation is used, start conditions become very simple to generate, even when many constraints are used.

\section{Results}

\subsection{Drilled Holes}

There are many examples of drilled holes to be found in the machined objects which we regularly use to test our algorithms. Often these holes are filled before scanning in order to reduce the problems discussed earlier and because the supporting surfaces are often the most important ones to parameterize.

\subsubsection{Primitive Extraction}

Primitives were extracted using the RANSAC algorithm as discussed earlier. The exact locations of the holes are not known beforehand but in some cases we know the inter-hole distance and can use this as a method of assessing positional accuracy. Mean error in radius estimation for 25 drilled holes was found to be $0.165 \mathrm{~mm}$ (standard deviation $0.128 \mathrm{~mm}$ ) using holes ranging from $5 \mathrm{~mm}$ to $10 \mathrm{~mm}$ radius. On our calibrated drilled block this gave an average inter-hole positional error of $0.235 \mathrm{~mm}$, over estimates of 24 difference positions.

\subsubsection{Optimization}

We used a calibration block drilled with 10 holes (example data from which is shown in figure 2) where the inter-hole distances are known, to apply geometric constraints. This gave us an improvement in positional accuracy, positional errors became $0.134 \mathrm{~mm}$ on average (standard deviation $0.120 \mathrm{~mm}$ ), with the 
same set of 24 observations. Radius estimates for the calibration block improved slightly, with the average error in radius estimate now being $0.163 \mathrm{~mm}$.

\subsection{Slots}

\subsubsection{Primitive Extraction}

Slot lengths were found to have the highest degree of variation, with an average error of $2.061 \mathrm{~mm}$ (standard deviation $1.565 \mathrm{~mm}$ ) over 72 examples. Slot angles were taken using pairs of slot sides. Mean slot angle error was $0.850^{\circ}$ (standard deviation $0.769^{\circ}$ ) over some 36 samples. Slot depths were harder to collect although of those that were possible, the angular error - that is the difference in surface normal between the slot bottom and the supporting surface - was found to be under $5^{\circ}\left(4.89^{\circ}\right)$ although no claims are made for this result as its stability is poor. Slot widths could not be properly ascertained since the lines were not necessarily found to be parallel. Variations in error were between 1 and $3 \mathrm{~mm}$.

\subsubsection{Optimization}

After optimization the slot results improved significantly in terms of angular error, since it was used as a geometric constraint and was based on the very accurate estimate of the supporting surface normal, and width variations. The width of all slots found in each of the slotted objects was constrained to be the same and the chromosome representing it consisted of the normal direction of the slot, 3 point chromosomes defining the positions and one width which was applicable to all of the slots. This degree of constraint produced slot widths that had a consistent error which is clearly systematic. Over 8 sets of data (that is 8 sets of three slots) the slot direction normal error was $0^{\circ}$ with a width error of $0.5 \mathrm{~mm}$ (standard deviation $0.04 \mathrm{~mm}$ ). The removal of this error is discussed in section 5 .

\subsection{Example timings}

For primitive extraction the average time for processing is under 1 minute on a $200 \mathrm{MHz}$ UltraSparc (average load) when analysing 8000 line hypotheses per dataset and 3000 hole hypotheses. This number of hypotheses is relatively generous however. When the Genocop III algorithm is used the average time for a run is between 5 minutes and 10 minutes depending upon the initialisation used (random or directed). Random initialisation is often achievable but not desirable as the position of the early reference solutions in the search space are very far away from the desirable solutions. This can effectively double processing time, or more, depending on the number of parameters that are used.

\section{Conclusions}

In this paper we have posed the question of whether it is possible to extract and optimize parametric models of machined artifacts from poor quality range data. We have shown that this is not only possible but that it may be done 
in reasonable amount of time. In previous work $[8,9]$ we have shown that optimal surface fitting under geometric constraints for large and well formed surfaces was feasible with an evolutionary algorithm. In this paper we have demonstrated that the method is equally applicable to model fitting for poor data. The preprocessing of the data using ransac to extract primitives has also greatly improved the speed of the algorithm both in terms of segmentation and approximation.

Good results have been derived from data that is very difficult to analyse under normal circumstances.

Since the data in these examples suffers from particularly acute sampling problems it is necessary to do further modelling of the error characteristics in order to remove the systematic errors that we have discovered. These almost certainly come from either the quantisation of the sampling space or from the surface characteristics of the data. In order to improve this aspect of the approach more work needs to be done to design a better model of where real discontinuities occur relative to our data discontinuity points.

We intend to implement a constraints-based model desription language that will be used in all phases of the optimization, from primitive finding through to the final optimization using genocop. This will have the added bonus that the $a$ priori geometric constraints can be used in all of the sub-parts of the scheme instead of just the final optimization. Finally, we envisage a full processing system that will be able to extract (i.e. segment), fit and finally optimize very complex models consisting of many surfaces and artifacts such as those described. High level descriptions will be pieced together by the user from a central library and the processing pipeline will create the CAD model, complete with optimized parameterisations.

\section{Acknowledgements}

The work presented in this paper was funded by a UK EPSRC grant GR/H86905.

\section{References}

[1] D. Eggert, A. W. Fitzgibbon, R. B. Fisher. "Simultaneous registration of multiple range views for use in reverse engineering", Proc. Int. Conf. on Pat. Recog., pp 243-247, Vienna, Aug. 1996.

[2] N. Werghi, R.B. Fisher, A. Ashbrook, C.Robertson "Modelling Objects Having Quadric Surfaces Incorporating Geometric Constraints." Proc. ECCV'98, pp.185-201, Freiburg, Germany, June 1998.

[3] Z. Michalewicz, Genetic Algorithms + Data Structures = Evolution Programs, Third Edition, Springer, 1996.

[4] A. Hoover, G. Jean-Baptiste, X. Jiang, P. J. Flynn, H. Bunke, D. Goldgof, K. Bowyer, D. Eggert, A. Fitzgibbon, R. Fisher, "An Experimental Comparison 
of Range Segmentation Algorithms", IEEE Trans. Pat. Anal. and Mach. Intel., Vol 18(7), pp673-689, July 1996

[5] M. Gen and R. Cheng, "A Survey of Penalty techniques in Genetic Algorithms", In Proceedings of the IEEE International Conference on Evolutionary Computation 1996, 1996.

[6] Z. Michalewicz and N. Attia, "Evolutionary Optimization of Constrained Problems", in , Proceedings of the 3rd Annual Conference on Evolutionary Programming, San Diego, CA, 1994, pages 98-108. World Scientific.

[7] Z. Michalewicz, D. Dasgupta, R. G. Le Riche and M. Schoenauer, "Evolutionary algorithms for constrained engineering problems", special issue on Genetic Algorithms and Industrial Engineering, ed. M. Gen, G.S.Wasserman and A. E. Smith, International Journal of Computers and Industrial Engineering, 1996.

[8] C. Robertson, D. Corne, R. B. Fisher, N.Werghi, A.Ashbrook, "Investigating Evolutionary Optimisation of Constrained Functions to Capture Shape Descriptions from Range Data", Proc. 3rd On-line World Conference on Soft Computing (WSC3) also in Advances in Soft Computing - Engineering Design and Manufacturing, eds. Roy, Furuhashi and Chawdhry, SpringerVerlag, 1998.

[9] C. Robertson, R. B. Fisher, N. Werghi, A. Ashbrook. "An Evolutionary Approach to Fitting Constrained Degenerate Second Order Surfaces", to be published in the Proceedings of EvoIASP'99, Sweden (28th May 1999), eds. Poli, Voigt, Cagnoni, Corne, Smith and Fogarty, Springer-Verlag Berlin, 1999.

[10] N. F. Attia, New Methods of Constrained Optimization Using Penalty Functions, Ph.D Thesis, Essex University, United Kingdom, 1985.

[11] R. C. Bolles and M. A. Fischler, "Random Sample Consensus: A Paradigm for Model Fitting with Applications to Image Analysis and Automated Cartography", Technical Note 213, Artificial Intelligence Center, SRI International, Menlo Park, California, 1980. 